\title{
Two-week virtual reality training for dementia: Single-case feasibility study
}

\author{
Daniel McEwen, MSc $;{ }^{1-2}$ Anne Taillon-Hobson, PT; ${ }^{1}$ Martin Bilodeau, PhD $;^{1-3}$ Heidi Sveistrup, PhD ${ }^{2-3}$ Hillel \\ Finestone, MDCM, FRCPC ${ }^{1,3-5 *}$ \\ ${ }^{1}$ Bruyère Research Institute, Ottawa, Ontario, Canada; Schools of ${ }^{2}$ Rehabilitation Sciences and ${ }^{3}$ Human Kinetics and \\ ${ }^{4}$ Faculty of Medicine, University of Ottawa, Ottawa, Ontario, Canada; ${ }^{5}$ Department of Physical Medicine and Reha- \\ bilitation, Elisabeth Bruyère Hospital, Ottowa, Ontario, Canada
}

\begin{abstract}
Persons with dementia (PWD) are known to have difficulty with participation and focus during physical activity. Virtual reality (VR) offers a unique medium for motor learning but has only been used previously for cognitive assessment for PWD. Our study had two objectives: (1) investigate the feasibility and safety of an exercise-based VR training program in PWD, and (2) investigate its effects on balance and mobility. The intervention consisted of daily ( $5 \mathrm{~d} / \mathrm{wk}, 1 \mathrm{~h}$ each) VR training sessions for $2 \mathrm{wk}$ for a single research participant. Clinical balance and mobility measures were assessed 1 wk prior to, during, $1 \mathrm{wk}$ following, and $1 \mathrm{mo}$ after the intervention. Postintervention interviews provided qualitative feedback from the participant and his caregivers. Results indicate that VR training is feasible, safe, and enjoyable for PWD. However, balance and mobility measures were unaffected. VR training is well tolerated in a single research participant with dementia and is an engaging medium for participation in exercise.
\end{abstract}

Key words: balance, dementia, exercise, games, intervention, rehabilitation, single-subject design, training, virtual reality, walking.

\section{INTRODUCTION}

Dementia is a growing healthcare concern. The 2012 World Health Organization report on dementia estimated a worldwide prevalence of persons with dementia (PWD) at 35.6 million. This number is expected to double by 2030 and more than triple by 2050 . Exercise has been shown to be physically and cognitively beneficial for numerous populations, including those with dementia. A recent systematic review on physical activity for PWD reported randomized controlled trials that assessed a wide range of exercise interventions including aerobics, stretching, and strengthening [1]. Selected outcomes of these trials included improved walking speed [2], improved strength and flexibility [3], and improved performance on the Timed "Up and Go" test (TUG) [4].

Virtual reality (VR) training has been shown to be an effective, motivating, and safe training tool when used alone or as an adjunct to conventional rehabilitation. Several studies have found objective improvements in clinical balance and mobility outcome measures in a variety of populations [5-8]. VR study protocols involve the adjustment of VR training parameters in order to meet the changing needs and rehabilitation goals of each individual patient. Task difficulty should reflect the skill

\footnotetext{
Abbreviations: $\mathrm{BBS}=$ Berg Balance Scale, IREX $=$ interactive rehabilitation exercise, $\mathrm{OSSm}=$ modified Ottawa Sitting Scale, $\mathrm{PWD}=$ persons with dementia, $\mathrm{SD}=$ standard deviation, TMWT $=$ Two-Minute Walk Test, TUG = Timed "Up and Go" test, $\mathrm{VR}=$ virtual reality.

"Address all correspondence to Hillel Finestone, MDCM, FRCPC; Bruyère Research Institute, 43 Bruyère St, Ottawa, Ontario, Canada, K1N 5C8; 613-562-6094; fax: 613-562-6312. Email: hfinestone@bruvere.org http://dx.doi.org/10.1682/JRRD.2013.10.0231
} 
level of the performer [9] in order to maximize performance outcome. Therefore, close monitoring of task difficulty while a patient is engaged in a stimulating environment will help ensure an appropriate level of challenge while maintaining engagement, motivation, and enjoyment. VR training is particularly well suited to allow such a versatile and engaging environment [10].

VR technology has been used with PWD for cognitive assessments [11] and cognitive training [12]. Multiple studies found that using virtual environments is feasible with PWD without problems of cybersickness or disorientation (e.g., Flynn et al. [13]). PWD are known to experience balance and mobility issues [14], and since previous studies have reported improvements in balance and mobility in other populations following an intensive VR training program, similar results could be expected in the PWD population. Because PWD can experience difficulty with exercise program adherence [15], the engaging and motivating nature of VR training may help alleviate this problem. The purpose of this study was to provide initial evidence (case study) of the effect of an intensive VR training program on balance and mobility in a veteran with dementia.

The study was conducted on an outpatient basis over an 8 wk period. Because it was unknown whether this methodology and protocol would be feasible for an outpatient client with dementia and his caregivers, this case study had two objectives: (1) to explore the feasibility and safety of an intensive, outpatient-based, VR training exercise program for a veteran with dementia and (2) to assess the effects of VR training on balance and mobility in this individual.

\section{METHODS}

\section{Participant}

Mr. YZ is a $78 \mathrm{yr}$ old, right-handed veteran who met the criteria for vascular dementia. His score on the Montreal Cognitive Assessment [16], a well-accepted screening test of cognition, was 12/30 (less than 26 is abnormal; 12 is extremely low). At the time of the study, Mr. YZ was taking daily doses of acetylsalicylic acid $(81 \mathrm{mg})$ in addition to Aricept via a patch. Bilateral subcortical microvascular disease was noted on brain imaging. Two years prior to his entry into this study, he presented with a right cortical ischemic stroke affecting his right occipital lobe. He had no known stroke risk factors.
Physical examination revealed a left visual field deficit. Mr. YZ ambulated independently with occasional handheld guidance for cueing. He intermittently complained of right tibiofemoral joint pain. However, no palpable warmth, erythema, or limited range of motion were noted on assessment. He demonstrated full range of motion and strength within normal limits for his age in both upper limbs. His scores for arm, leg, and posture subscales of the Chedoke-McMaster Stroke Assessment, a reliable and valid measure used to assess physical impairment and disability in clients with stroke and other neurological impairment [17], were 6, 5, and 5, respectively, and were used as standard measures of his functional status. Each dimension is measured on a 7-point scale ranging from 1 (total assistance) to 7 (safely independent). His scores indicate he requires supervision.

\section{Research Design}

A single-subject design was used to identify changes in balance and functioning during, immediately following, and 1 mo after a daily, 2 wk long VR training program (Table). The participant's power of attorney provided written informed consent in accordance with the research ethics board at the Bruyère Research Institute. Mr. YZ was accompanied at all sessions by one of his permanent caregivers.

Table.

Illustration of timing of outcome assessments.

\begin{tabular}{lll}
\hline \multicolumn{1}{c}{ Phase } & \multicolumn{1}{c}{ Frequency/Duration } & Tests \\
\hline Preintervention & 3 sessions in 1 wk & BBS \\
& & TUG \\
& & TMWT \\
& & OSSm \\
Intervention & 5 times per week for 2 wk & BBS \\
& & TMWT \\
Postintervention & 3 sessions in 1 wk & BBS \\
& & TUG \\
& & TMWT \\
1 mo Follow-Up & 1 session & OSSm \\
& & BBS \\
& & TUG \\
& & TMWT \\
& & OSSm
\end{tabular}

$\overline{\text { BBS }}=$ Berg Balance Scale (score out of 56; lower scores indicate lower function), OSSm = modified Ottawa Sitting Scale (score out of 24; lower score indicates lower function), TUG = Timed "Up and Go" test (lower score indicaties better mobility), TMWT $=$ Two-Minute Walk Test (maximum distance walked in $2 \mathrm{~min}$; higher scores indicate higher function). 


\section{Intervention}

The VR intervention was delivered using the interactive rehabilitation exercise (IREX) software (GestureTek; Toronto, Canada), which involves the use of green screen technology. Mr. YZ stood in front of a 50 in. television located $10 \mathrm{ft}$ away that displayed his image with the use of a camera, immersed in five different virtual environments in which he interacted with virtual objects (Appendix, available online only). The applications were chosen to train his standing balance and were administered in the same order each day. Each session lasted approximately $1 \mathrm{~h}$ with an average of $25 \mathrm{~min}$ of VR exercise time. The remaining time was spent resting and explaining how to play the upcoming game.

Because of the progressive nature of training programs, a baseline soccer game with a consistent difficulty level allowed for assessment of improvements with interacting with the VR system. Mr. YZ thus completed $1 \mathrm{~min}$ of a standardized soccer application at the beginning of each training session. The scores on this baseline game provided an overall skill evaluation. On the soccer goaltending application, balls saved on the right and left sides were counted to determine the effect of Mr. YZ's visual field deficit on his performance and to assess any related performance changes as the training progressed.

In order to ensure safety from falls or stumbles, Mr. YZ wore a physiotherapy belt and was monitored during all sessions by a registered kinesiologist. The VR applications were selected in order to elicit specific movements that would challenge balance, including reaching beyond arm's length, weight shifting, and lateral stepping. Rest was given as needed to prevent fatigue and to retain focus.

\section{Feasibility}

To the best of our knowledge, this is the first implementation of a VR exercise training protocol with a person with dementia. Therefore, factors that facilitated or hindered feasibility were identified through interviews with caregivers following each session and at the end of the intervention trial. Positive events during training as well as intervention-related adverse events were also documented.

Open-ended interview questions prepared by the research team were used to obtain feedback from the family and care providers regarding the safety and usefulness of the intervention as well as their perception of Mr. YZ's enjoyment during the VR sessions. The exit interview was conducted by a third party who was uninvolved with the research project. Responses were recorded and transcribed for extraction of major themes.

\section{Clinical Outcome Measures}

Four clinical outcome measures, TUG [18], Berg Balance Scale (BBS) [19], a modified Ottawa Sitting Scale (OSSm) [20], and the Two-Minute Walk Test (TMWT) [21], were used to measure functional balance and motor performance.

The BBS is a 14-item validated standardized test with a maximum score of 56 . It measures static, dynamic, adaptive, and anticipatory components of balance [22]. Community-dwelling elderly people and PWD have been reported to score $54.0 \pm 3.0$ [23] and $47.5 \pm 16.9$ [24], respectively. The TUG is a validated test of functional movement requiring rising from a chair, walking $3 \mathrm{~m}$, negotiating a turn, and returning to sit back in the chair. It is reliable for use with people with dementia with scores ranging from 17.1 to $24.7 \mathrm{~s}$ [25-26]. A score of $13.5 \mathrm{~s}$ or more has been shown to indicate a high risk of falls in community-dwelling older adults [27]. Functional endurance measured with the TMWT documented the maximum distance Mr. YZ could safely walk in $2 \mathrm{~min}$. The mean distance recorded for PWD in respite care is $32.2 \pm$ $15.7 \mathrm{~m} \mathrm{[28]} \mathrm{and} \mathrm{for} \mathrm{retirement} \mathrm{home} \mathrm{dwelling} \mathrm{older} \mathrm{adults}$ is $150.4 \pm 23.1 \mathrm{~m}$ [29]. The OSSm, with six tasks graded on a scale of 1 to 4 for a maximum score of 24, was used as an indicator of static and dynamic sitting balance.

All measures were recorded on Monday, Wednesday, and Friday during the week prior to and the week following the VR training sessions as well as once at the 1 mo follow-up (Table). Also, each day immediately before completing the VR training session, Mr. YZ performed the BBS and the TMWT. All clinical outcome measures were administered by the same experienced registered physiotherapist.

\section{Analysis}

When appropriate, means and standard deviations (SDs) as well as individual scores are reported and describe performance in outcome measures. Statistical analysis using the 2-SD band method [30] was performed for both the BBS and the TMWT. Results are considered to be statistically significant if two consecutive data points are outside the 2-SD band. If the values at followup remained outside the 2-SD band, significance was considered to be maintained. 


\section{RESULTS}

\section{Qualitative Results}

Mr. YZ attended all VR training sessions and was always a cooperative participant. He was able to complete the entire game set in each session provided he had sufficient rest between the applications. He did not experience any negative effects of the VR such as cybersickness, dizziness, loss of balance, or falls. Mr. YZ intermittently understood the information provided about the nature of VR, the equipment setup, and his role in playing the VR applications. He followed instructions within the limits of his concentration abilities throughout the exercise sessions.

Difficulties encountered with implementing VR training with $\mathrm{Mr}$. YZ arose from his memory deficits and his difficulty concentrating on the tasks at hand. He frequently did not remember what applications he had completed in earlier sessions, and thus daily instructions were required and there was no opportunity to progress task complexity. Mr. YZ would often stop in the middle of an application because he was distracted. He was not always actively engaged and thus could not always provide a full effort during the training. His visual field deficit was manifested by a frequent inability to attend to activities occurring on his left side.

During a postintervention interview with his caregivers, family members reported Mr. YZ had greater concentration when performing activities at home such as dressing in the morning, with less time required on training days. Family members noted relatively higher levels of physical activity, and this gave them confidence to encourage Mr. YZ to perform other physical activities at home. More energy and interest in doing physical activities throughout the day, for example walking the dog, were also commented upon. The family also reported $\mathrm{Mr}$. $\mathrm{YZ}$ was more engaged in the VR games than during his home exercises.

\section{Quantitative Results}

\section{Clinical Outcome Measures}

No changes were found in the clinical measures of balance and mobility for the BBS, TMWT, or OSSm.

BBS scores (Figure 1(a)) fluctuated from an average of $50.0 \pm 1.0$ (pre-VR) to $48.6 \pm 2.3$ (post-VR) and 50.0 (no SD because only 1 measure) (1 mo follow-up).
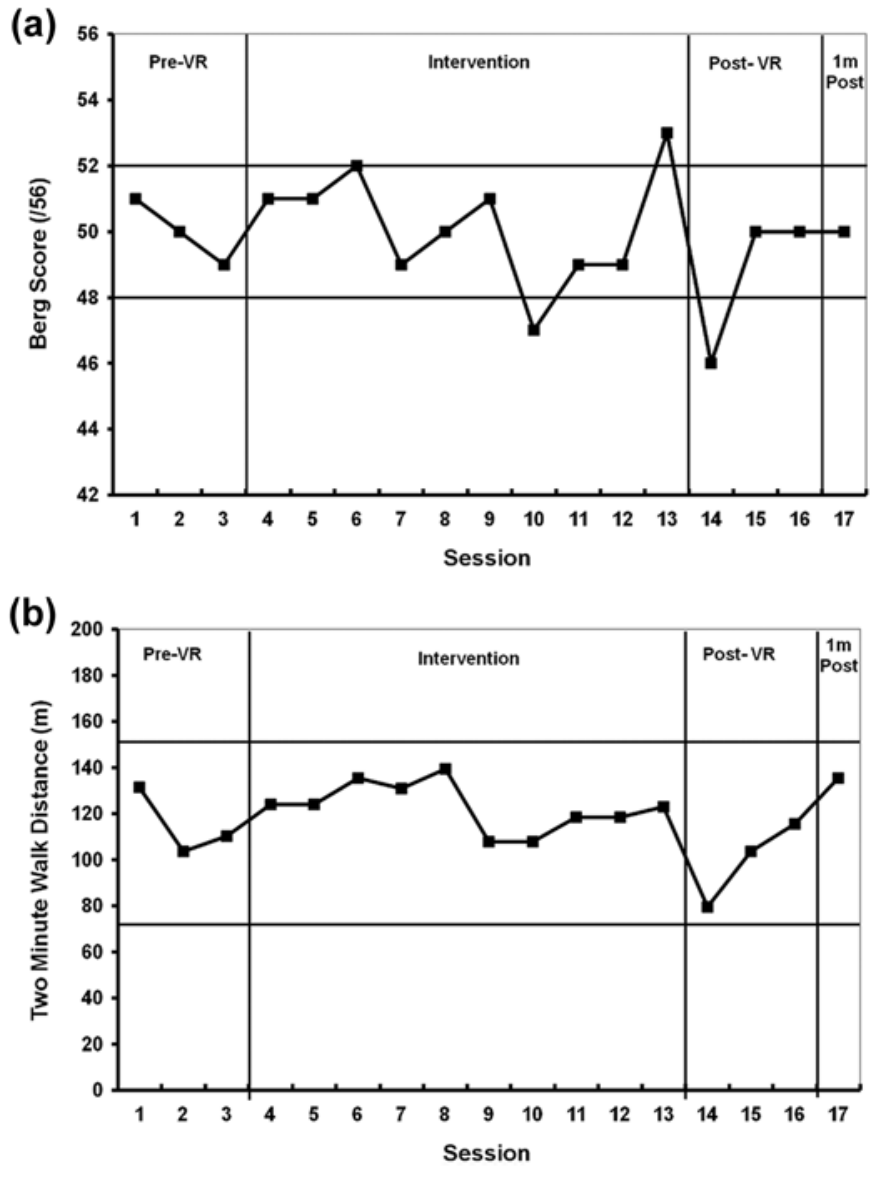

Figure 1.

Visual representation of (a) Berg Balance Scale and (b) TwoMinute Walk Test. Solid horizontal lines indicate \pm 2 standard deviations from the mean of pre-VR scores. Berg Balance Scale is score out of 56; lower scores indicate lower function. Normative value for age-matched controls is 53/56 [31]. TwoMinute Walk Test is maximum distance walked in 2 min; higher scores indicate higher function. Normative value for age matched controls (70-79 yr) is $191.5 \mathrm{~m}$ [32]. VR = virtual reality.

The average distances covered in the TMWT (Figure 1(b)) were $115.1 \pm 14.5 \mathrm{~m}$ (pre-VR), $122.9 \pm 10.5 \mathrm{~m}$ (intervention phase), $99.6 \pm 18.3 \mathrm{~m}$ (post-VR), and $135.5 \mathrm{~m}$ (1 mo follow-up). There were no significant differences between the preintervention, postintervention, and 1 mo follow-up measurements.

There was a marked decline in both the BBS and TMWT scores on the first post-VR session (session 14). Session 14 was done on a Monday morning. The participant had been busy with family outings over the weekend and was complaining about right knee pain at the start of 
session 14. This may have decreased his gait speed and weight-bearing ability, lowering his scores on the BBS and the TMWT distance on this particular day.

Visual analysis of the TUG (Figure 2) indicated a significant change between the postintervention and the 1 mo follow-up assessments. At the pre- and postintervention, scores were respectively $26.3 \mathrm{~s}$ and $28.7 \mathrm{~s}$, while at $1 \mathrm{mo}$ follow-up, the mean time improved to $14.3 \mathrm{~s}$. The OSSm scores (Figure 2) did not change during the study.

\section{Performance Outcome Measures}

Data from the baseline soccer application played at the beginning of every session show that Mr. YZ was able to learn and improve on the VR applications (Figure 3). Comparing the first five with the last five sessions, there was an increased number of saves on his left side, the side with the visual field deficit. There was no change in the number of saves on his right side.

\section{DISCUSSION}

This study investigated the feasibility, safety, and effectiveness of an intensive 10-session VR intervention program for an individual with dementia. Our results suggest that VR is a feasible and safe activity for PWD. Significant objective improvements in the clinical measures of balance and mobility were not found, but postintervention interviews with the participant and his caregiver revealed that the intervention was an enjoyable experience, which seemed to have a motivating effect on his participation in activities at home.

VR training was shown to be feasible since Mr. YZ attended every session and participated in the full training session without any adverse events (falls, dizziness, cybersickness, etc.). However, Mr. YZ demonstrated frequent limitations in concentration and occasionally forgot how to play the games. These factors made it difficult to confidently progress the level of difficulty of the games in order to ensure an adequate effort level to allow for measurable performance improvements. Ideally, any future modifications to the games of the IREX or any other VR system should allow for adjustments of game parameters to meet the attention requirements of a client with dementia or other cognitive/attentional impairments.

This study was conducted on an "outpatient" basis, but not within an outpatient physiotherapy setting. From a health professional human resource perspective, the fre-

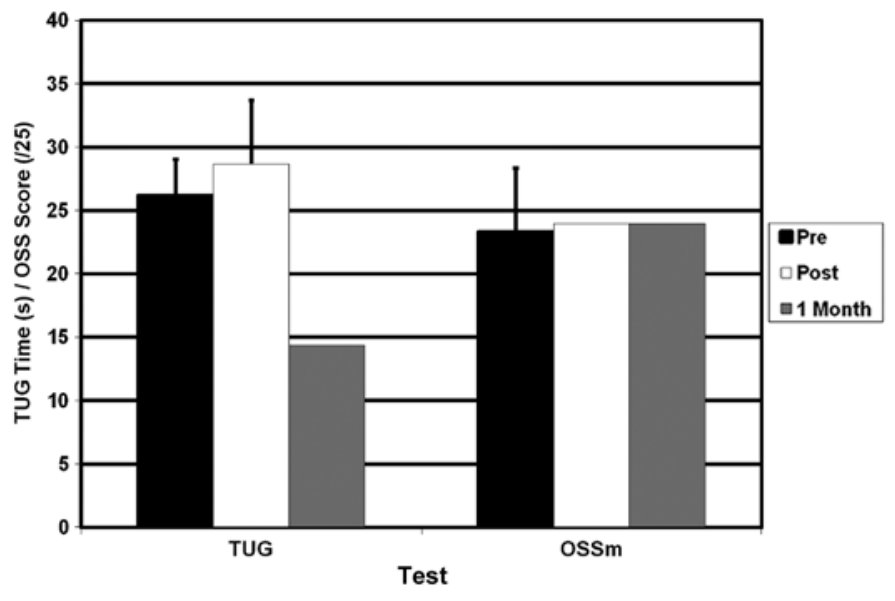

Figure 2.

Mean scores for clinical measures completed at pretest, posttest, and 1 mo follow-up only. Bars represent standard deviation. Absence of standard deviation bar in posttest modified Ottawa Sitting Scale (OSSm) is due to same score at all three posttest sessions, while 1 mo follow-up measures for OSSm and Timed "Up and Go" test (TUG) are from single test session. No normative values available for OSSm. Normative value for nondisabled age-matched controls on TUG is $8.39 \pm 1.36 \mathrm{~s}$ [33].

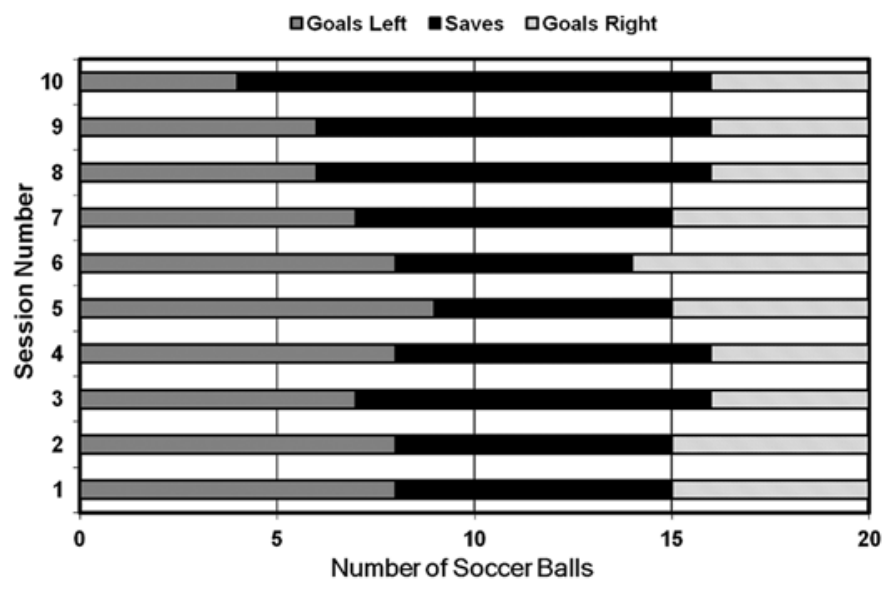

Figure 3.

Scores in soccer baseline set. Same $60 \mathrm{~s}$ application with 20 total soccer balls was used prior to every virtual reality training session.

quency of appointments required by this type of intensive VR exercise training program could be a challenge. Because of the frequency of the appointments (daily) over 
a relatively short period of time (2 wk), suitable staffing support should be considered when planning future VR intervention programs with a larger sample size.

Although this 2 wk intensive VR training program was shown to be feasible, it was not effective in improving clinical balance and mobility for Mr. YZ. Compared with nondisabled individuals of similar age, Mr. YZ's clinical measures were impaired at baseline and thus there was potential for improvements with intervention. Although the TUG did show significant improvement between postintervention and 1 mo follow-up, there was no improvement between the pre-post time points, suggesting the improvement may not be directly due to the intervention. The lack of intervention-dependent improvements (pre-post comparisons) in the clinical measures likely reflects a confounding effect of the cognitive impairment on motor function. For example, Mr. $\mathrm{YZ}$ often did not use the full range of his arm movements or challenge his base of support by leaning or stepping despite repeated encouragement and demonstrations. He also appeared to forget how to play the games in between sessions, and attempts to achieve "just right" challenge [9] proved difficult. The 1 mo improvement in the TUG may be an indirect effect of the VR intervention. Specifically, the care providers indicated that witnessing $\mathrm{Mr}$. YZ's performance in the VR environment facilitated their encouragement for activity at home. Mr. YZ did, however, improve his task performance in the virtual soccer application. The decrease in the number of goals, particularly on the left side, indicates that he was able to learn the game, as indicated by a decreased need for instructions. The participant had left visual field deficits. We speculate that game participation increased scanning ability to the left, leading to a decrease in the number of goals on the left (Figure 3).

\section{CONCLUSIONS}

VR exercise using the IREX system was found to be an enjoyable, safe, and feasible intervention for a single research participant with dementia. Physical benefits from training were reported from family members, including increased reports of energy and more involvement in activities of daily living at home. However, objective clinical measures of balance and mobility failed to demonstrate significant improvements following this short intervention. The VR technology was able to encourage activity in our study participant (despite his low cognition) and he was able to succeed at the VR games. These observations suggest that VR game play provides an opportunity to increase leisure activity and challenge cognitive skills; aspects that should be explored in greater detail. Finally, although we failed to see any improvements on physical outcome measures, future studies are required to assess whether intervention programs with longer exposure and at higher intensity are feasible with PWD and whether they would result in measurable improvement on game scores (e.g., soccer) as well as balance and mobility outcomes.

\section{ACKNOWLEDGMENTS}

\section{Author Contributions:}

Study concept and design: D. McEwen, A. Taillon-Hobson, M. Bilodeau, H. Sveistrup, H. Finestone.

Implementation of intervention: D. McEwen, A. Taillon-Hobson. Acquisition of data: A. Taillon-Hobson, D. McEwen.

Analysis and interpretation of data: D. McEwen, A. Taillon-Hobson, M. Bilodeau, H. Sveistrup, H. Finestone.

Drafting of manuscript: D. McEwen, A. Taillon-Hobson,

M. Bilodeau, H. Sveistrup, H. Finestone.

Critical revision of manuscript: D. McEwen, A. Taillon-Hobson, M. Bilodeau, H. Sveistrup, H. Finestone.

Obtained funding: H. Finestone, M. Bilodeau, H. Sveistrup.

Financial Disclosures: The authors have declared that no competing interests exist. GestureTek was not involved in the study design; collection, analysis, and interpretation of data; writing of the report; or decision to submit the report for publication.

Funding/Support: This material was based on work supported by a research grant from The Heart and Stroke Foundation of Ontario, Centre for Stroke Recovery; the Dr. Tony Hakim Special Project Award; and a generous donation by Elisabeth and Tony Graham. D.M. holds a Queen Elisabeth II Ontario Graduate Scholarship in Science \& Technology and an Excellence Scholarship from the University of Ottawa. Additional Contributions: Sincere thanks goes to our participant and his caregivers for their hard work and dedication throughout the entirety of the study.

Institutional Review: The Bruyère Research Institute Ethics Committee approved this study. Our participant's power of attorney signed an informed consent form.

Participant Follow-Up: There has been ongoing follow-up with the participant and his family of the study results.

\section{REFERENCES}

1. Potter R, Ellard D, Rees K, Thorogood M. A systematic review of the effects of physical activity on physical functioning, quality of life and depression in older people with 
dementia. Int J Geriatr Psychiatry. 2011;26(10):1000-11. [PMID:21905096]

http://dx.doi.org/10.1002/gps.2641

2. Toulotte C, Fabre C, Dangremont B, Lensel G, Thévenon A. Effects of physical training on the physical capacity of frail, demented patients with a history of falling: A randomised controlled trial. Age Ageing. 2003;32(1):67-73. [PMID:12540351] http://dx.doi.org/10.1093/ageing/32.1.67

3. Baum EE, Jarjoura D, Polen AE, Faur D, Rutecki G. Effectiveness of a group exercise program in a long-term care facility: A randomized pilot trial. J Am Med Dir Assoc. 2003;4(2):74-80. [PMID:12807578] http://dx.doi.org/10.1016/S1525-8610(04)70279-0

4. Santana-Sosa E, Barriopedro MI, López-Mojares LM, Pérez M, Lucia A. Exercise training is beneficial for Alzheimer's patients. Int J Sports Med. 2008;29(10):84550. [PMID:18401810] http://dx.doi.org/10.1055/s-2008-1038432

5. Saposnik G, Levin M; Outcome Research Canada (SORCan) Working Group. Virtual reality in stroke rehabilitation: A meta-analysis and implications for clinicians. Stroke. 2011;42(5):1380-86. [PMID:21474804] http://dx.doi.org/10.1161/STROKEAHA.110.605451

6. Brien M, Sveistrup H. An intensive virtual reality program improves functional balance and mobility of adolescents with cerebral palsy. Pediatr Phys Ther. 2011;23(3): 258-66. [PMID:21829120] http://dx.doi.org/10.1097/PEP.0b013e318227ca0f

7. Thornton M, Marshall S, McComas J, Finestone H, McCormick A, Sveistrup H. Benefits of activity and virtual reality based balance exercise programmes for adults with traumatic brain injury: Perceptions of participants and their caregivers. Brain Inj. 2005;19(12):989-1000. [PMID:16263641] http://dx.doi.org/10.1080/02699050500109944

8. Subramanian SK, Lourenço CB, Chilingaryan G, Sveistrup H, Levin MF. Arm motor recovery using a virtual reality intervention in chronic stroke: Randomized control trial. Neurorehabil Neural Repair. 2013;27(1):13-23.

[PMID:22785001]

http://dx.doi.org/10.1177/1545968312449695

9. Guadagnoli MA, Lee TD. Challenge point: A framework for conceptualizing the effects of various practice conditions in motor learning. J Mot Behav. 2004;36(2):212-24. [PMID:15130871] http://dx.doi.org/10.3200/JMBR.36.2.212-224

10. Sveistrup H. Motor rehabilitation using virtual reality. J Neuroeng Rehabil. 2004;1(1):10. [PMID:15679945] http://dx.doi.org/10.1186/1743-0003-1-10

11. Cushman LA, Stein K, Duffy CJ. Detecting navigational deficits in cognitive aging and Alzheimer disease using virtual reality. Neurology. 2008;71(12):888-95. [PMID:18794491] http://dx.doi.org/10.1212/01.wnl.0000326262.67613.fe

12. Man DW, Chung JC, Lee GY. Evaluation of a virtual reality-based memory training programme for Hong Kong Chinese older adults with questionable dementia: A pilot study. Int J Geriatr Psychiatry. 2012;27(5):513-20.

[PMID:21681818] http://dx.doi.org/10.1002/gps.2746

13. Flynn D, van Schaik P, Blackman T, Femcott C, Hobbs B, Calderon C. Developing a virtual reality-based methodology for people with dementia: A feasibility study. Cyberpsychol Behav. 2003;6(6):591-611. [PMID:14756925] http://dx.doi.org/10.1089/109493103322725379

14. Hauer K, Becker C, Lindemann U, Beyer N. Effectiveness of physical training on motor performance and fall prevention in cognitively impaired older persons: A systematic review. Am J Phys Med Rehabil. 2006;85(10): 847-57. [PMID:16998433] http://dx.doi.org/10.1097/01.phm.0000228539.99682.32

15. Forbes D, Forbes S, Morgan DG, Markle-Reid M, Wood J, Culum I. Physical activity programs for persons with dementia. Cochrane Database Syst Rev. 2008;16(3): CD006489. [PMID:18646158]

16. Nasreddine ZS, Phillips NA, Bédirian V, Charbonneau S, Whitehead V, Collin I, Cummings JL, Chertkow H. The Montreal Cognitive Assessment, MoCA: A brief screening tool for mild cognitive impairment. J Am Geriatr Soc. 2005;53(4):695-99. [PMID:15817019] http://dx.doi.org/10.1111/j.1532-5415.2005.53221.x

17. Gowland C, Stratford P, Ward M, Moreland J, Torresin W, Van Hullenaar S, Sanford J, Barreca S, Vanspall B, Plews N. Measuring physical impairment and disability with the Chedoke-McMaster Stroke Assessment. Stroke. 1993; 24(1):58-63. [PMID:8418551] http://dx.doi.org/10.1161/01.STR.24.1.58

18. Podsiadlo D, Richardson S. The timed "Up \& Go": A test of basic functional mobility for frail elderly persons. J Am Geriatr Soc. 1991;39(2):142-48. [PMID:1991946]

19. Blum L, Korner-Bitensky N. Usefulness of the Berg Balance Scale in stroke rehabilitation: A systematic review. Phys Ther. 2008;88(5):559-66. [PMID:18292215] http://dx.doi.org/10.2522/ptj.20070205

20. Thornton M, Sveistrup H. Intra- and inter-rater reliability and validity of the Ottawa Sitting Scale: A new tool to characterise sitting balance in acute care patients. Disabil Rehabil. 2010;32(19):1568-75. [PMID:20662547] http://dx.doi.org/10.3109/09638280903567893

21. Brooks D, Davis AM, Naglie G. The feasibility of sixminute and two-minute walk tests in in-patient geriatric rehabilitation. Can J Aging. 2007;26(2):159-62. 
JRRD, Volume 51, Number 7, 2014

\section{[PMID:17613447]}

http://dx.doi.org/10.3138/cja.26.2.009

22. Berg KO, Wood-Dauphinee SL, Williams JI, Maki B. Measuring balance in the elderly: Validation of an instrument. Can J Public Health. 1992;83(Suppl 2):S7-11. [PMID:1468055]

23. Steffen TM, Hacker TA, Mollinger L. Age- and genderrelated test performance in community-dwelling elderly people: Six-Minute Walk Test, Berg Balance Scale, Timed Up \& Go Test, and gait speeds. Phys Ther. 2002;82(2): 128-37. [PMID:11856064]

24. Miu, D., Szeto, S., Mak, Y. A randomised controlled trial on the effect of exercise on physical, cognitive and affective function in dementia subjects. Asian J Gerontol Gariatr. 2008;3:8-16.

25. Netz Y, Axelrad S, Argov E. Group physical activity for demented older adults feasibility and effectiveness. Clin Rehabil. 2007;21(11):977-86. [PMID:17984150] http://dx.doi.org/10.1177/0269215507078318

26. Kwak YS, Um SY, Son TG, Kim DJ. Effect of regular exercise on senile dementia patients. Int J Sports Med. 2008;29(6):471-74. [PMID:18050054] http://dx.doi.org/10.1055/s-2007-964853

27. Shumway-Cook A, Brauer S, Woollacott M. Predicting the probability for falls in community-dwelling older adults using the Timed Up \& Go Test. Phys Ther. 2000; 80(9):896-903. [PMID:10960937]

28. Pomeroy VM, Warren CM, Honeycombe C, Briggs RS, Wilkinson DG, Pickering RM, Steiner A. Mobility and dementia: Is physiotherapy treatment during respite care effective? Int J Geriatr Psychiatry. 1999;14(5):389-97. [PMID:10389044] http://dx.doi.org/10.1002/(SICI)10991166(199905)14:5<389::AID-GPS933>3.0.CO;2-8

29. Connelly DM, Thomas BK, Cliffe SJ, Perry WM, Smith RE. Clinical utility of the 2-minute walk test for older adults living in long-term care. Physiother Can. 2009; 61(2):78-87. [PMID:20190990] http://dx.doi.org/10.3138/physio.61.2.78
30. Nourbakhsh MR, Ottenbacher KJ. The statistical analysis of single-subject data: A comparative examination. Phys Ther. 1994;74(8):768-76. [PMID:8047564]

31. Ferri CP, Prince M, Brayne C, Brodaty H, Fratiglioni L, Ganguli M, Hall K, Hasegawa K, Hendrie H, Huang Y, Jorm A, Mathers C, Menezes PR, Rimmer E, Scazufca M; Alzheimer's Disease International. Global prevalence of dementia: A Delphi consensus study. Lancet. 2005; 366(9503):2112-17. [PMID:16360788] http://dx.doi.org/10.1016/S0140-6736(05)67889-0

32. Selman J, de Camargo A, Santos J, Lanza F, Dal Corso S. Reference equation for the two-minute walk test in adults and the elderly. Respir Care. 2014;59(4):525-30. [PMID:23920212]

33. Hofheinz M, Schusterschitz C. Dual task interference in estimating the risk of falls and measuring change: A comparative, psychometric study of four measurements. Clin Rehabil. 2010;24(9):831-42. [PMID:20562166] http://dx.doi.org/10.1177/0269215510367993

Submitted for publication October 27, 2013. Accepted in revised form March 26, 2014.

This article and any supplementary material should be cited as follows:

McEwen D, Taillon-Hobson A, Bilodeau M, Sveistrup H, Finestone H. Two-week virtual reality training for dementia: Single-case feasibility study. J Rehabil Res Dev. 2014;51(7):1069-76.

http://dx.doi.org/10.1682/JRRD.2013.10.0231

ResearcherID/ORCID: Daniel McEwen, MSc: L-53502014; Heidi Sveistrup, PhD: C-6616-2011; Martin Bilodeau, PhD: L-8588-2014

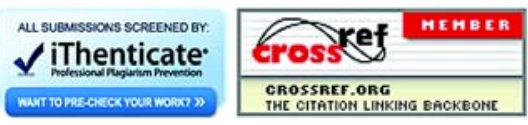

